Research Paper

\title{
Dioscin Induces Gallbladder Cancer Apoptosis by Inhibiting ROS-Mediated PI3K/AKT Signalling
}

Xiaoling Song ${ }^{1,2^{*}}$, Zheng Wang1, ${ }^{*}$, Haibin Liang ${ }^{1,2^{*}}$, Wenjie Zhang ${ }^{1 *}$, Yuanyuan Ye ${ }^{1,2}$, HuaiFeng Li ${ }^{1,2}$,

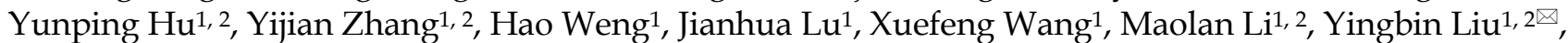
Jun $\mathrm{Gu}^{1 凶}$

1. Department of General Surgery and Laboratory of General Surgery, Xinhua Hospital affiliated to Shanghai Jiao Tong University School of Medicine, Shanghai, People's Republic of China;

2. Institute of Biliary Tract Disease, Shanghai Jiao Tong University School of Medicine, Shanghai, People's Republic of China.

* Xiaoling Song, Zheng Wang, Haibin Liang and Wenjie Zhang contributed equally to this work.

$凶$ Corresponding authors: Yingbin Liu email: liuybphd@126.com; Jun Gu email: gujun6666@sina.com

(c) Ivyspring International Publisher. This is an open access article distributed under the terms of the Creative Commons Attribution (CC BY-NC) license (https:// creativecommons.org/licenses/by-nc/4.0/). See http://ivyspring.com/terms for full terms and conditions.

Received: 2016.12.13; Accepted: 2017.03.25; Published: 2017.06.01

\begin{abstract}
Gallbladder cancer (GBC), highly aggressive form of cancer with an extremely poor prognosis, is the most common malignancy of the biliary tract. In this study, we investigated the effects of dioscin (DSN) on human GBC and the potential mechanisms underlying these effects. The results showed that DSN significantly inhibited GBC cell proliferation and migration. Moreover, DSN induced GBC cell apoptosis via mitochondrial dependent apoptotic signalling. Reactive oxygen species (ROS) and glutathione (GSH) levels were measured, and ROS scavengers completely inhibited DSN-induced apoptosis and migration, indicating that ROS play an essential role in GBC progression. Western blot analysis showed that AKT activity was significantly downregulated after DSN treatment, and that inhibition/ectopic expression of AKT enhanced/abolished DSN-induced apoptosis but not migration. Furthermore, we confirmed the relationship between ROS and the PI3K/AKT pathway and found that DSN induced apoptosis by regulating ROS-mediated PI3K/AKT signaling. Taken together, these findings indicate that DSN induces GBC apoptosis through inhibiting ROS-mediated PI3K/AKT signalling.
\end{abstract}

Key words: gallbladder cancer, apoptosis, dioscin, reactive oxygen species.

\section{Background}

GBC is the most common and aggressive biliary tract malignancy and the fifth gastrointestinal cancer. The only available curative treatment is complete surgical resection; however, only $10 \%$ of patients are eligible for surgery because of its asymptomatic characteristics and chemoresistance. As a result, the 5 -year survival rate for GBC remains between $0 \%$ and $10 \%$ in most reported series [4]. Additionally, among those patients who undergo surgical resection, recurrence rates remain high [4]. Therefore, novel and effective therapies are urgently needed.

Natural compounds, especially plant-derived compounds, have been widely used as therapeutic agents against cancer. Dioscin (DSN), a plant glucoside saponin, extracted from Dioscorea nipponica Makino and Dioscorea zingiberensis Wright, has been shown to exert many biological and pharmacological effects. Previous studies have shown that DSN has anti-fungal, anti-virus and hepatoprotective properties. DSN has recently attracted increasing amount of attention because of its anti-cancer effects on lung cancer, colon cancer, breast cancer and gastric cancer [9]. However, the effects of DSN on GBC has not been determined.

Reactive oxygen species (ROS) are a group of reactive, short-lived, oxygen-containing species, such as superoxide, singlet oxygen atoms, hydrogen peroxide, hydroxyl radicals and peroxyl radicals. ROS can activate intracellular signal transduction pathways in cancer, such as inflammation, cell cycle progression, apoptosis, migration and invasion. A previous investigation showed that DSN induced 
generation of ROS through mitochondria dysfunction [10]. Whether ROS generation exerts anti-cancer effects on GBC has not yet been illustrated. In this study, we investigated the effects of DSN on GBC cells and their potential mechanisms underlying the induction of GBC cell apoptosis and migration. Our results showed that DSN induced GBC cell apoptosis by regulating ROS-mediated PI3K/AKT signalling, thus we deduced DSN may represent a novel therapeutic intervention for GBC.

\section{Methods}

\section{Drugs and reagents}

DSN was purchased from Sigma-Aldrich (St. Louis, MO, USA); the purity was at least $98 \%$, as determined by high performance liquid chromatography. DSN was dissolved in dimethyl sulfoxide (DMSO) in a $100 \mathrm{mM}$ stock solution and stored at $-20^{\circ} \mathrm{C}$. The final DMSO concentration was less than $0.1 \%$. Cell Counting Kit-8 (CCK-8), Hoechst 33342 and Rhodamine 123 kits were purchased from Sigma-Aldrich. Annexin V/Propidium Iodide (PI) Apoptosis Kit was purchased from Invitrogen (Carlsbad, CA, USA). A pan-caspase inhibitor (Z-VAD-FMK) and PI3K inhibitor (LY294002) as well as GSH, NAC and GSH/GSSH and ROS detection kits were purchased from Beyotime Institute of Biotechnology Company (Suzhou, Jiangsu, China). All antibodies were purchased from Santa Cruz Biotechnology (Santa Cruz, CA, USA). All cell culture supplies were obtained from Invitrogen (Carlsbad, CA, USA).

\section{Cell culture}

The human GBC cell lines NOZ and SGC996 were purchased from the Cell Bank of the Type Culture Collection of the Chinese Academy of Sciences (Shanghai, Shanghai, China). NOZ cells were cultured in William's medium, and SGC996 cells were cultured in 1640 medium. All media were supplemented with $100 \mu \mathrm{g} / \mathrm{ml}$ streptomycin and 100 $\mathrm{U} / \mathrm{ml}$ penicillin and $10 \%$ foetal bovine serum. The cells were cultured at $37^{\circ} \mathrm{C}$ in a humidified incubator with $5 \% \mathrm{CO}_{2}$.

\section{Cell viability assay}

A CCK-8 assay was used to evaluate NOZ and SGC996 cell viability. Human kidney epithelial cells (293T) was used to determine the change of normal cells. Cells were seeded into 96-well plates at a density of 4000 cells/well and were cultured for approximately $24 \mathrm{~h}$. Various concentrations of DSN (0, $1,2,4,6,8 \mu \mathrm{M})$ were subsequently added, and the cells were incubated for $24 \mathrm{~h}, 48 \mathrm{~h}$ or $72 \mathrm{~h}$. After treatment, CCK-8 (10 $\mu 1)$ was added and incubated for
$3 \mathrm{~h}$ in the dark. Absorbance was measured at $450 \mathrm{~nm}$ using a microplate reader (Norcross, GA, USA). IC50 was measured by Graphpad Prism 5.

\section{Colony formation assay}

SGC996 and NOZ cells were seeded into 6-well plates with DSN $(0,2,4,6 \mu \mathrm{M})$ for 15 days. Then, the cells were fixed with $10 \%$ formalin and stained with $0.1 \%$ crystal violet (Sigma-Aldrich, St. Louis, MO, USA). After washing, the plates were dried and the colonies (with more than 50 cells) were observed under a microscope.

\section{Annexin V/PI staining assay for apoptosis}

SGC996, NOZ and 293T cells were treated with DSN for 48h. After centrifugation (1500rpm, 5min), the cells were combined with $1 \times$ Annexin $\mathrm{V}$ binding buffer and then incubated with 5ul Annexin V and PI at $37^{\circ} \mathrm{C}$ for $30 \mathrm{~min}$ in the dark. Cell apoptosis was measured using flow cytometry.

\section{Hoechst 33342 staining}

SGC996 and NOZ cells were treated with DSN $(0,2,4,6 \mu \mathrm{M})$ for $48 \mathrm{~h}$. After the cells were fixed with 1 $\mathrm{ml}$ methanol/acetic acid (3:1) and stained with $5 \mu \mathrm{g} / \mathrm{ml}$ Hoechst 33342, a fluorescence microscope was used to analyse the morphological changes.

\section{Mitochondrial membrane potential $(\Delta \Psi \mathrm{m})$ assay}

Cells were treated with DSN $(0,2,4,6 \mu \mathrm{M})$ for 48 $\mathrm{h}$, collected and washed with cold PBS. Then, the cells were incubated with Rhodamine 123 in a 5\% $\mathrm{CO}_{2}$ incubator at $37^{\circ} \mathrm{C}$ for $20 \mathrm{~min}$ in the dark. Finally, the cells were analysed by flow cytometry.

\section{Transwell migration analysis}

To prevent effects of proliferation and apoptosis, we chose the indicated concentrations $(0,0.5,1,2 \mu \mathrm{M})$ for subsequent assays. After the cells were resuspended, $200 \mu \mathrm{l}$ of medium containing $2 \times 10^{5}$ cells with different treatment was added into the upper well and incubated for 24-72h. Images of the stained cells were captured by microscopy.

\section{ROS and GSH detection}

Intracellular ROS generation was assessed using a ROS assay kit, and GSH generation was assessed using a GSH/GSSH assay kit according to the manufacturer's instructions.

\section{Plasmid transfection}

Two eukaryotic expression vectors containing cDNA encoding wild-type (WT) and constitutively active (CA) mouse AKT and an empty vector (pcDNA 3.1) were purchased from Era Biotech (Shanghai, 
Shanghai, China). Cells were seeded on 24-well plates and transfected for $24 \mathrm{~h}$ using Viafect transfection reagent, according to the manufacturer's protocol.

\section{Western blot analysis}

Proteins were separated using 10\% sodium dodecyl sulfate polyacrylamide gel electrophoresis (SDS-PAGE) and then transferred to polyvinylidene difluoride (PVDF) membranes. The membranes were blocked for $1 \mathrm{~h}$ at $37^{\circ} \mathrm{C}$ and incubated with primary antibodies against Bcl-2, Bax, cleaved caspase9, cleaved caspase3, cleaved poly(ADP ribose) polymerase (PARP), PI3K, pAKT, p-AKT and GAPDH overnight at $4^{\circ} \mathrm{C}$. Next, the membranes were incubated with secondary antibodies for $1 \mathrm{~h}$ at $37^{\circ} \mathrm{C}$. Proteins were observed using a Gel Doc 2000 (Berkeley, California, USA).

\section{In vivo tumour xenograft study}

All animal treatments were carried out in accordance with the National Institutes of Health Guide for the Care and Use of Laboratory Animals, and approved by the Institutional Animal Care and Use Committee of Shanghai Jiaotong University. Male nude mice (aged 4-6 weeks, weighting 18-22 g) were purchased from Shanghai SLAC Laboratory Animal Co Ltd (Shanghai, Shanghai, China). The animals were housed at $25^{\circ} \mathrm{C} \pm 2{ }^{\circ} \mathrm{C}$ at a relative humidity of $70 \% \pm 5 \%$ under natural light/dark conditions for 1 week and were allowed free access to food and water. $\mathrm{NOZ}$ cells (at a density of $1 \times 10^{6}$ cells in $0.2 \mathrm{ml}$ ) were injected into the right axilla of each mouse. Twenty-four hours later, the mice were randomly divided into 3 groups (control, $5 \mathrm{mg} / \mathrm{kg}, 10 \mathrm{mg} / \mathrm{kg}$ ). Mice received DSN at the appropriate dose $(0,5$ or $10 \mathrm{mg} / \mathrm{kg}$ ) every 3 days for up to 25 days. On day 26, the animals were sacrificed, and their tumours were dissected and weighed.

\section{HE staining and Immunohistochemistry}

After the mice were sacrificed, their tumours, livers and spleens were resected and immediately fixed in $10 \%$ formalin, embedded in paraffin, cut into $5 \mathrm{~mm}$ sections and mounted on slides. The expression patterns of PI3K and p-AKT were analysed using immunohistochemical (IHC) streptavidin-peroxidase staining.

\section{Statistical analysis}

All experiments were performed at least 3 times, and the results are expressed as the means \pm standard deviations unless otherwise stated. Student's t-test was used to compare differences between the treated groups and the corresponding control groups; $p<0.05$ was considered statistically significant.

\section{Results}

\section{DSN inhibits GBC cell proliferation and migration}

CCK-8 assay and colony formation assays were performed to determine the effects of DSN on GBC cell proliferation. As shown in Figure 1A-1B, NOZ and SGC996 cell proliferation was significantly inhibited by DSN in a dose-dependent manner, with an IC50 value of $4.465 \mu \mathrm{M}$ (NOZ) and $5.049 \mu \mathrm{M}$ (SGC996), and the numbers and sizes of the colonies treated with DSN were substantially smaller than those of the control group. Migration was evaluated by transwell migration analysis. We chose concentrations $(0,0.5,1,2 \mu \mathrm{M})$ that would not affect cell viability. As shown in Figure 1C, DSN repressed NOZ and SGC996 cell migration in a dose-dependent manner. Therefore, DSN inhibits GBC cell proliferation and migration.

\section{DSN induces GBC cell apoptosis}

We investigated the effects of DSN on GBC cell apoptosis by flow cytometry and Hoechst 33342 staining. Compared with control group, DSN-treated cells exhibited significant chromatin condensation and fragmentation, and the percentage of early and late apoptosis cells were strikingly elevated in a dose-dependent manner (Figure 2A-2B). As no normal gallbladder cells were available, we used human kidney epithelial cells (293T) to determine whether normal cells undergo apoptosis following DSN treatment [1]; CCK-8 and flow cytometry assays revealed that DSN-treated 293T cells did not undergo apoptosis( Figure 2C -2D).

\section{DSN induces mitochondrial dependent apoptosis in NOZ and SGC996 cell}

Mitochondria regulates the mitochondrial permeability transition and the release of toxic components, such as caspases to regulate apoptosis. The loss of $\Delta \Psi \mathrm{m}$ was indicated by a decrease in Rhodamine 123 fluorescent staining intensity. Compared with control group, DSN-treatment increased numbers of Rhodamine 123-negative cells (Figure 3A). Moreover, DSN treatment upregulated the levels of cleaved caspase PARP, 3 and 9, which may have facilitated mitochondrial dependent apoptosis (Figure 3B). To confirm these results, we evaluated cell viability after DSN treatment in the presence or absence of Z-VAD-FMK, a caspase inhibitor. As shown in Figure 3D, Z-VAD-FMK abolished DSN-induced GBC cell cytotoxity. Together, these results indicate that DSN induces mitochondrial dependent apoptosis in NOZ and SGC996 cell. 
A
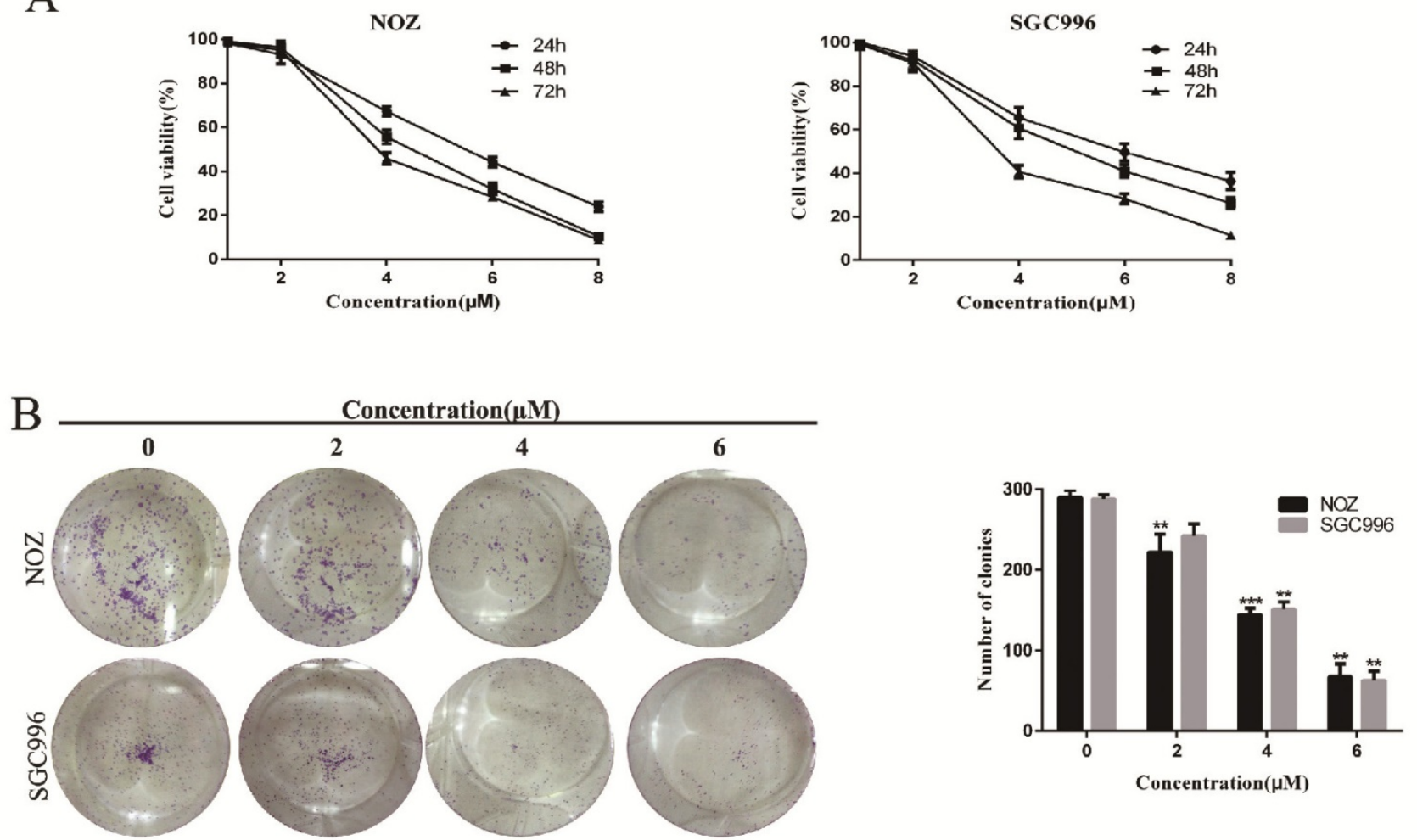

$\mathrm{C}$
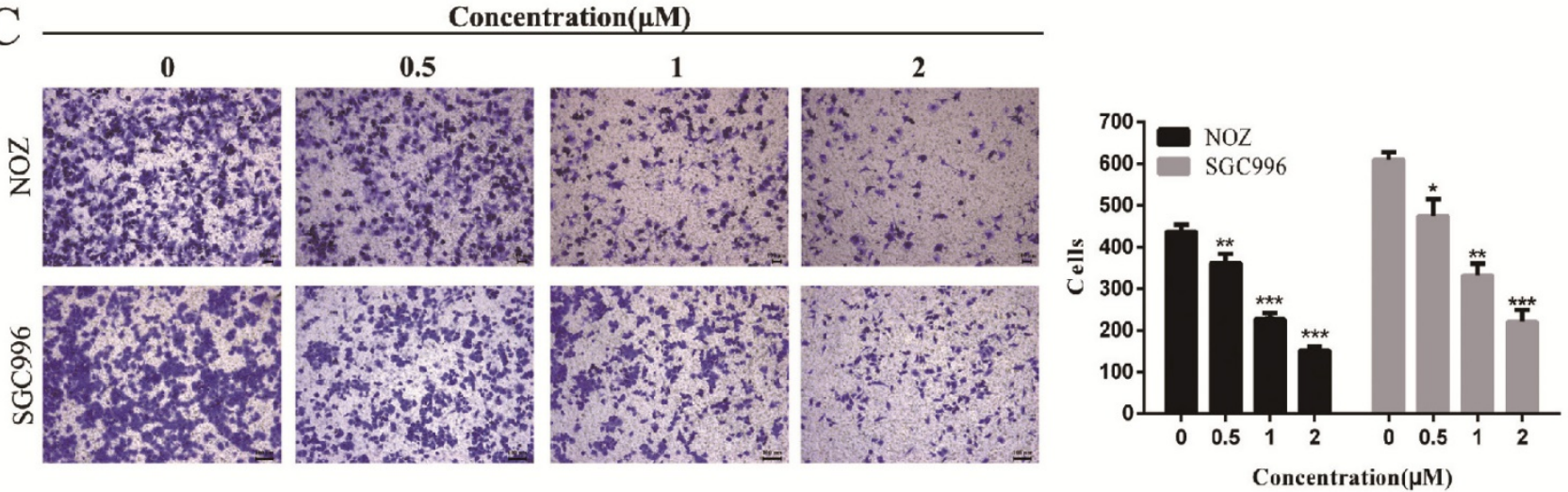

Figure 1. DSN inhibits GBC cell proliferation and migration. A) NOZ and SGC996 cells were treated with DSN (0, 1, 2, 4, 6, 84M) for $24 \mathrm{~h}, 48 \mathrm{~h}$ and $72 \mathrm{~h}$. CCK-8 assays were carried out to assess proliferation. B) DSN suppressed NOZ and SGC996 cell colony formation. Cells were exposed to DSN (0,2, 4, 6uM) and were allowed to form colonies for 15 days. C) The effects of DSN on GBC cell migration were assessed by transwell migration analysis. All data are presented as the means \pm standard deviations, and each experiment was repeated 3 times. Significant differences compared with the control are indicated by $*_{p}<0.05$, $* * p<0.01$, and $* * * p<0.001$.

\section{ROS accumulation is associated with DSN-induced apoptosis and migration inhibition}

Many studies have revealed that ROS accumulation can induce cell death in many types of cancers after treatment with anti-cancer drugs. Therefore, we investigated whether ROS are associated with DSN-induced apoptosis. We detected intracellular ROS generation following different DSN treatment intervals, and we found that ROS generation was most significant at $4 \mathrm{~h}$ of treatment (Figure S1A). As shown in Figure 4A, increasing amounts of ROS were generated in a dose-dependent manner at lower concentrations than at higher concentrations, as higher doses were ineffective at inducing ROS because of their strong cytotoxic effect. Consistent with these results, fluorescence analysis by microscopy also demonstrated that ROS generation was dose-dependent (Figure S1B). GSH is the major cellular ROS-scavenger, treatment strategies inducing deceases in the level of reduced GSH may have a 
profound effect on cell survival and drug sensitivity by altering the ability of cells to detoxify ROS [15]. Thus, we investigated whether DSN-induced apoptosis is related to GSH levels. After treatment with DSN for $48 \mathrm{~h}$, dose-dependent decreases in intracellular GSH levels were observed, as shown in Figure 4B. Moreover, we treated GBC cell in the presence or absence of the ROS scavengers NAC and $\mathrm{GSH}$, and then assessed intracellular ROS production and cytotoxicity. As shown in Figure 4C $-4 \mathrm{E}$, NAC and GSH abolished DSN-induced ROS accumulation and cytotoxicity. Furthermore, NAC and GSH also abolished DSN-induced mitochondrial dependent apoptosis (Figure 4E -4F, Figure S1C). Consistent with the findings of previous studies, we found that ROS accumulation was related to DSN-induced migration inhibition (Figure S2A-S2B).

Collectively, our results showed that DSN-induced apoptosis and migration are related to mitochondrial ROS accumulation.
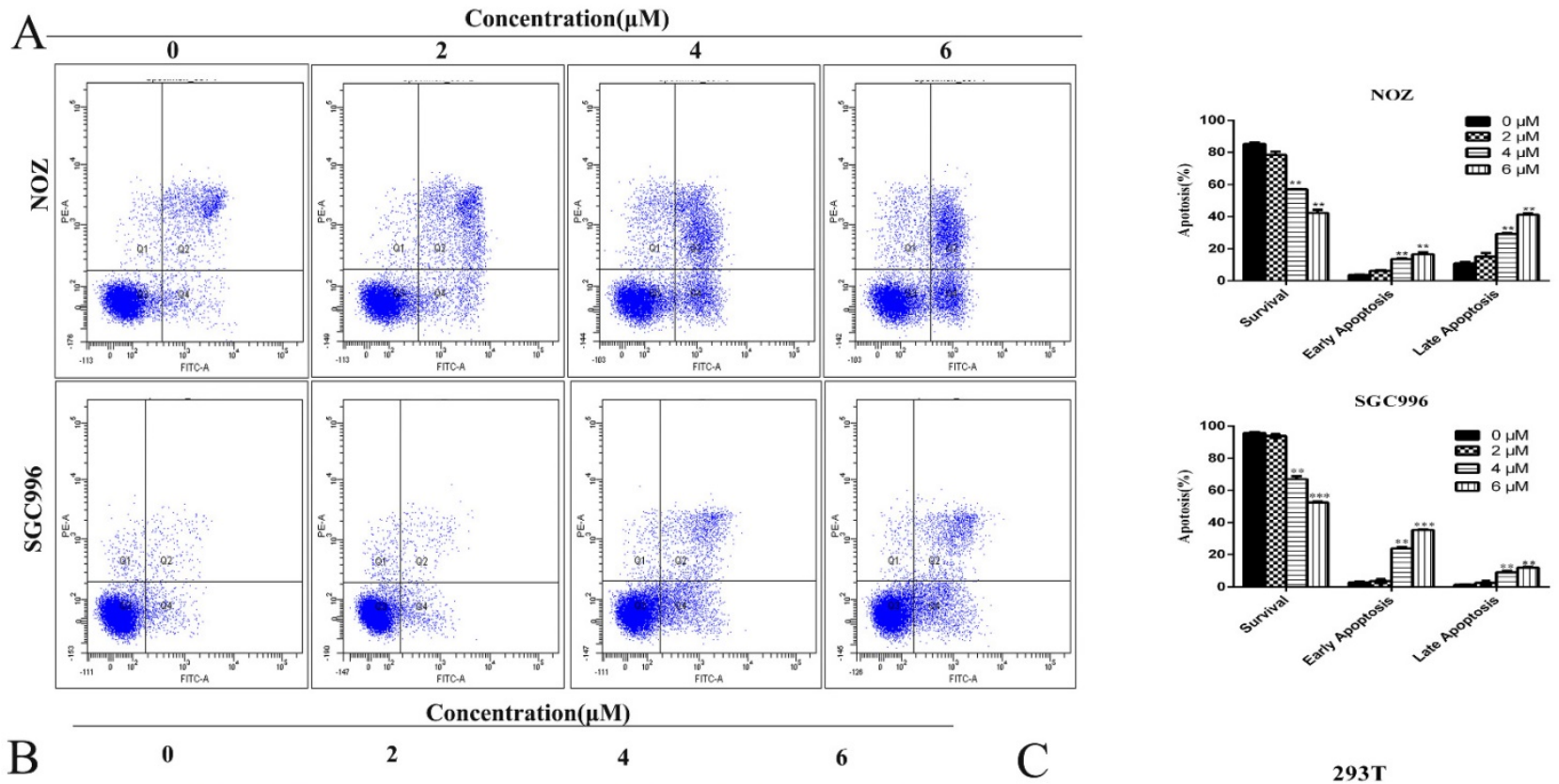

$\mathrm{B}$

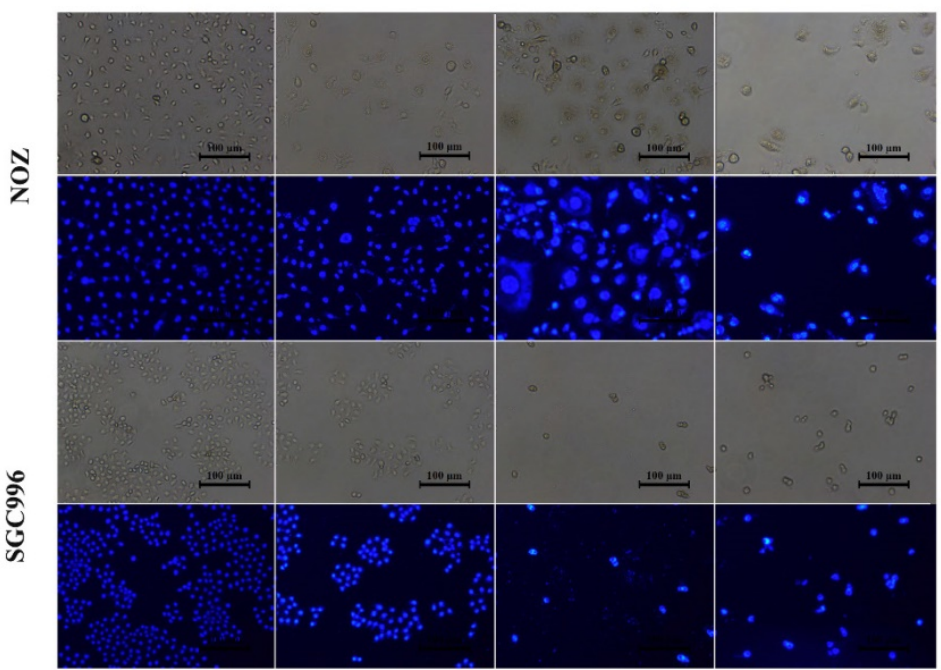

C

D
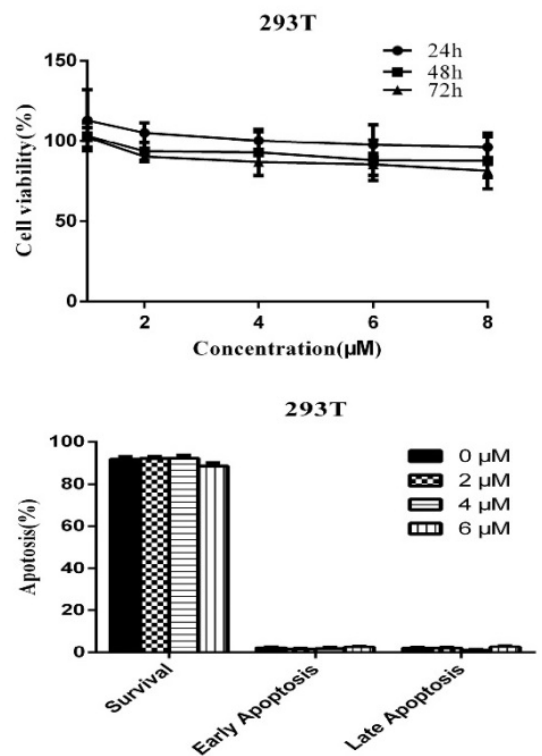

Figure 2. DSN induces GBC cell apoptosis. A) NOZ and SGC996 cells were treated with DSN for $48 \mathrm{~h}$. Apoptosis was analysed by flow cytometry. B) NOZ and SGC996 cells were exposed to DSN for $48 \mathrm{~h}$, and the nuclear morphological changes associated with apoptosis were evaluated by Hoechst33342 staining. C-D) $293 \mathrm{~T}$ cell viability and apoptosis were evaluated by CCK-8 analysis and flow cytometry. All data are presented as the means \pm standard deviations, and each experiment was repeated 3 times. Significant differences compared with the control are indicated by $*_{p}<0.05$, $* * p<0.01$, and $* * * p<0.001$. 
A

B

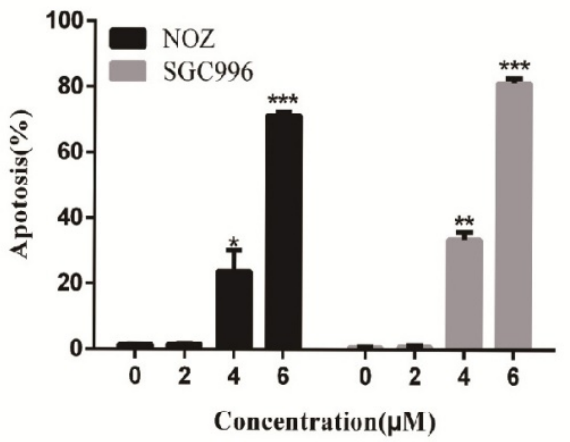

C

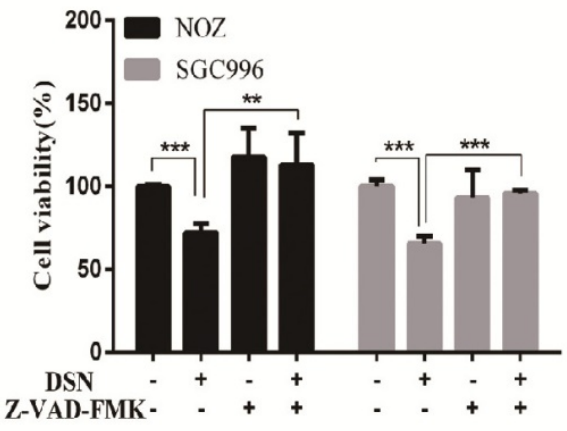

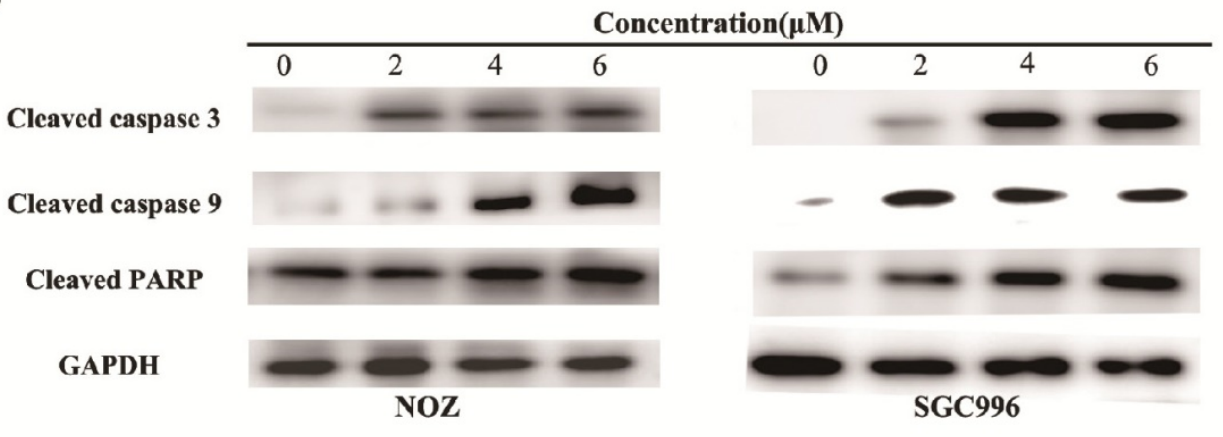

D DSN -

Z-VAD-FMK -
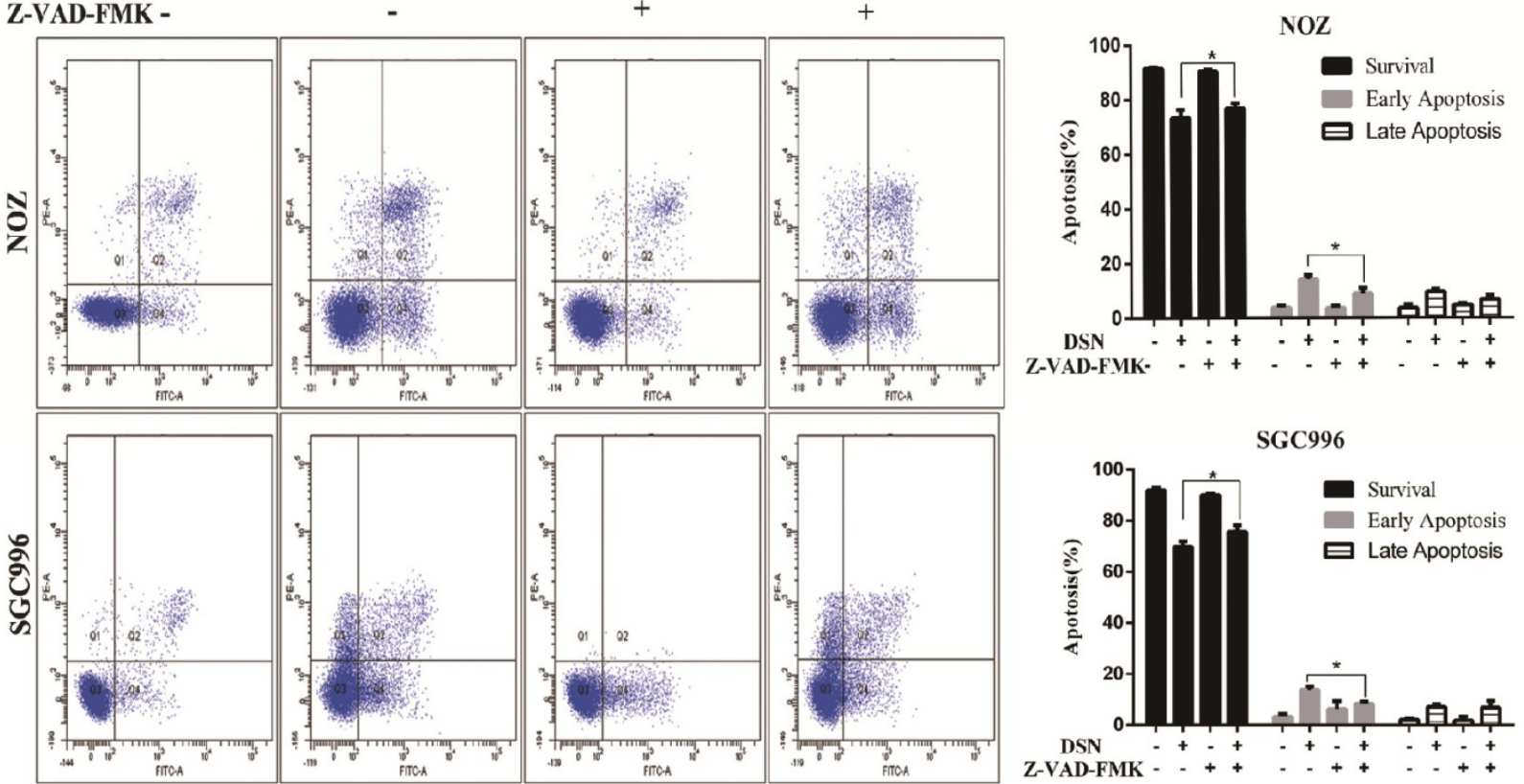
Z-VAD-FYK- $-++-++\cdots$

Figure 3. DSN induces mitochondrial dependent apoptosis in NOZ and SGC996 cell. A) NOZ and SGC996 cells were treated with DSN and stained with Rhodamine 123. Cells with high $\Delta \Psi_{\mathrm{m}}$ are marked "survival", and those with low $\Delta \Psi_{\mathrm{m}}$ are marked "apoptosis". B) Apoptosis-related protein expression in NOZ and SGC996 cells was analysed by Western blot analysis. GAPDH was used as a loading control. C-D) After treatment with DSN in the presence or absence of Z-VAD-FMK, GBC cell viability and apoptosis ware evaluated by CCK-8 analysis and flow cytometry.

\section{DSN induces GBC cell apoptosis by inhibiting the PI3K/AKT signalling pathway}

The PI3K/AKT pathway is one of the major signalling pathways associated with the growth of various tumours, and it is also associated with cancer progression and invasion. To determine whether the
PI3K/AKT signalling pathway is associated with DSN-induced apoptosis, we estimated the effects of DSN on PI3K/AKT-related protein expression by western blot analysis. As shown in Figure 5A, DSN significantly inhibited PI3K/AKT signalling. To further confirm whether DSN-induced GBC apoptosis is mediated by Akt activity inhibition, we next 
transfected wild-type AKT (WT-AKT) and constitutively active AKT (CA-AKT) into GBC cells. As shown in Figure 5C, S3A-3C, ectopic expression of AKT (Figure 5B) abolished DSN-induced apoptosis and cytotoxicity. After treatment with DSN in the presence or absence of LY294002, a PI3K/AKT inhibitor, we found that LY294002 significantly enhanced DSN-induced cell death (Figure 5D-5E, S4A-S4B). Furthermore, we found that the PI3K/AKT signalling pathway is not associated with DSN-induced migration inhibition (Figure S5A-S5D). Together, these results indicate that the PI3K/AKT signalling pathway is associated with DSN-induced apoptosis but not migration.

\section{DSN induces GBC cell apoptosis by regulating ROS-mediated PI3K/AKT signalling}

Our previous results showed that DSN-induced GBC apoptosis is related to ROS accumulation and PI3K/AKT signalling pathway inhibition. To confirm the relationship between ROS and the PI3K/AKT signalling pathway, we examined GBC cells in the presence or absence of NAC or GSH. As showed in Figure 6A, NAC and GSH enhanced PI3K, p-AKT and AKT expression. However, ectopic AKT and LY294002 had no effect on ROS generation (Figure 6C-6D). Thus, we believe that DSN induces GBC cell apoptosis by regulating ROS-mediated PI3K/AKT signalling not AKT-mediated ROS accumulation.
A

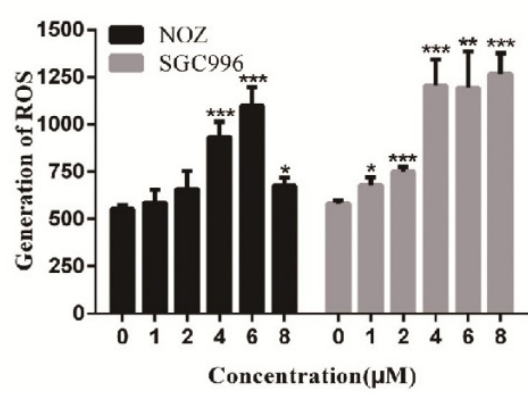

$\mathrm{D}$

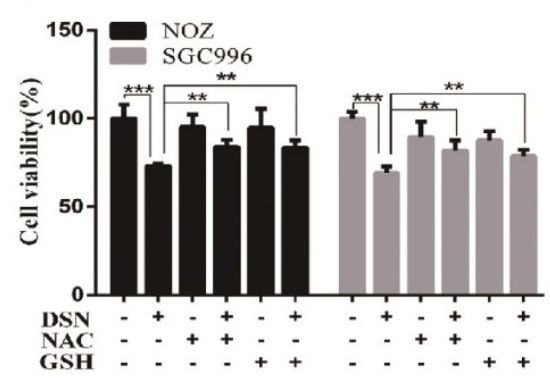

B

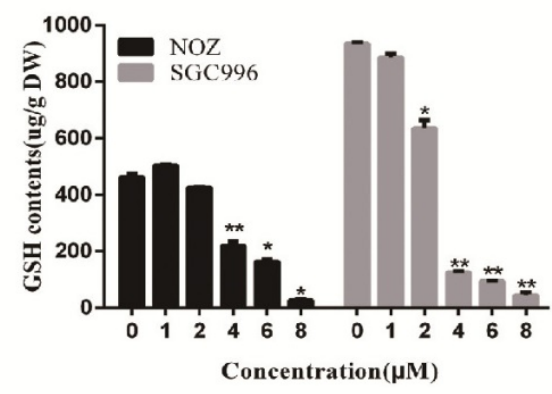

$\mathrm{E}$

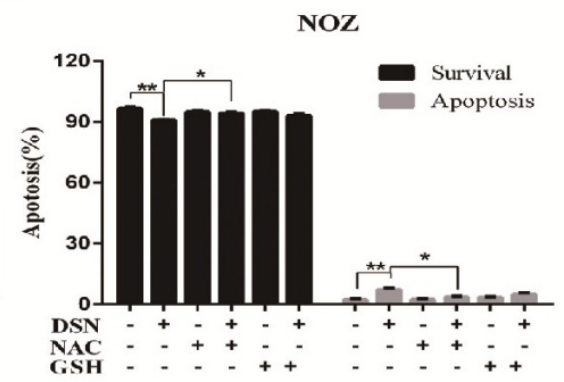

$\mathrm{C}$

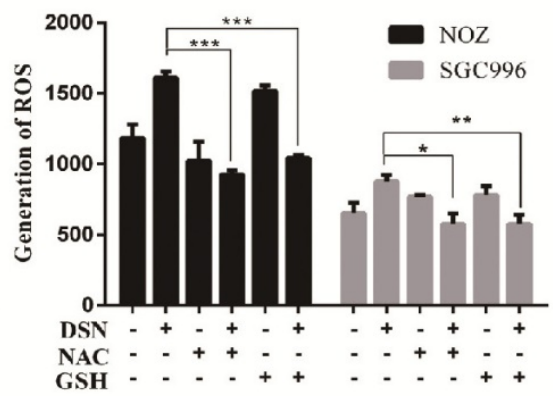

$\mathrm{F}$
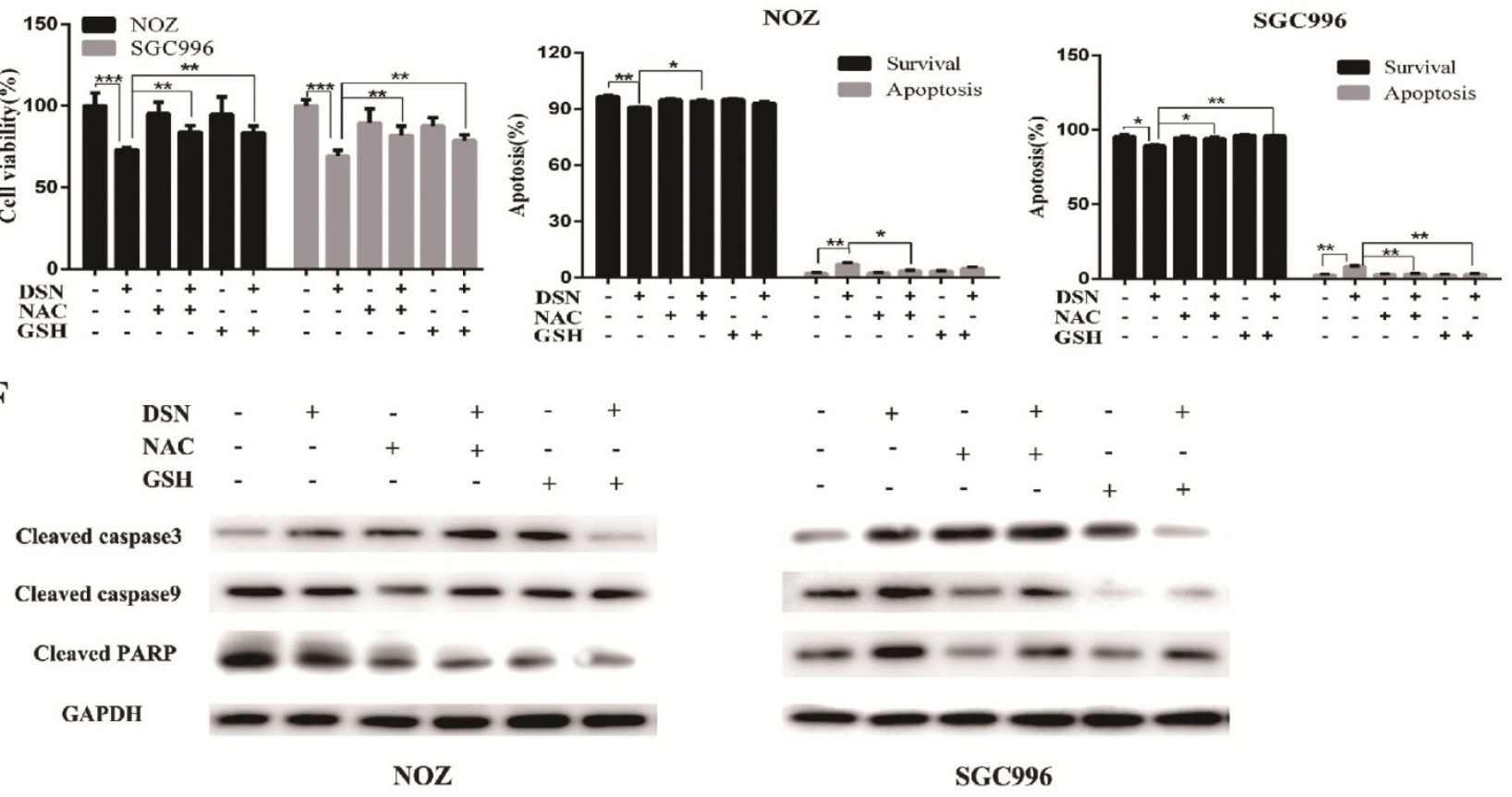

SGC996

Figure 4. ROS accumulation is associated with DSN-induced apoptosis and migration inhibition. A) NOZ and SGC996 cells were treated with various

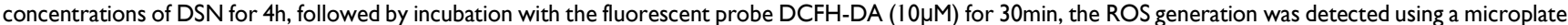
reader. B) GSH generation was determined in the absence or presence of $5 \mathrm{mM} \mathrm{NAC}$ or $5 \mathrm{mM}$ GSH for $1 \mathrm{~h}$. C-D) ROS generation and cell viability were determined in the absence or presence of $5 \mathrm{mM} \mathrm{NAC}$ or $5 \mathrm{mM} \mathrm{GSH}$ for $1 \mathrm{~h}$. E) NOZ and SGC996 cells were treated in the absence or presence of NAC and GSH for $24 \mathrm{~h}$. Apoptosis was analysed by flow cytometry. F) Apoptosis-related protein expression in NOZ and SGC996 cells was analysed by western blot. GAPDH was used as a loading control. 


\section{Concentration $(\mu M)$}

A

Bcl-2

0

2

4

6

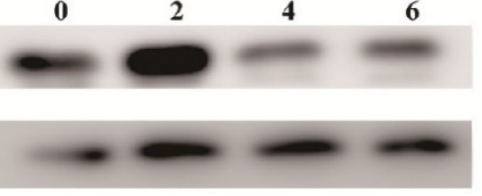

Bad

Bax

p-AKT

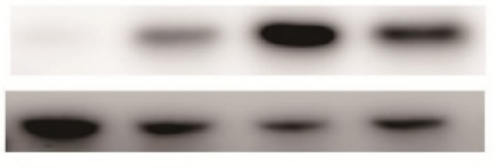

AKT

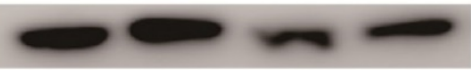

PI3K

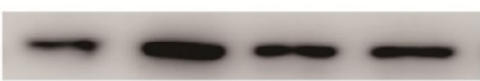

GAPDH

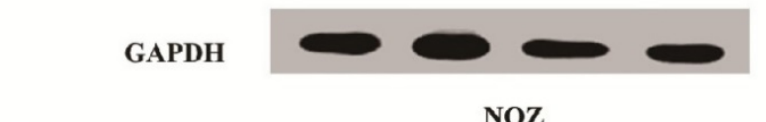

NOZ

$\begin{array}{lllll}0 & 2 & 4 & 6 \\ & & & & \\ & & & & \end{array}$
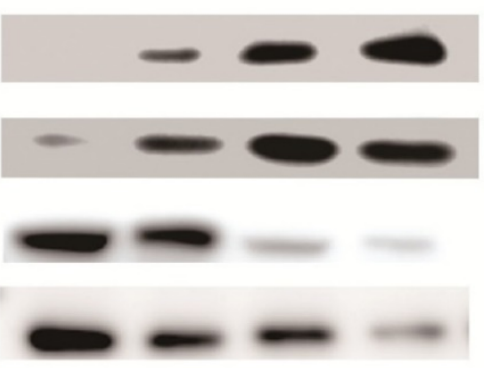

B

$\begin{array}{lllllll}\text { DSN } & - & - & - & + & + & + \\ \text { pcDNA3.1 } & + & - & - & + & - & - \\ \text { CA-AKT } & - & + & - & - & + & - \\ \text { WT-AKT } & - & - & + & - & - & +\end{array}$
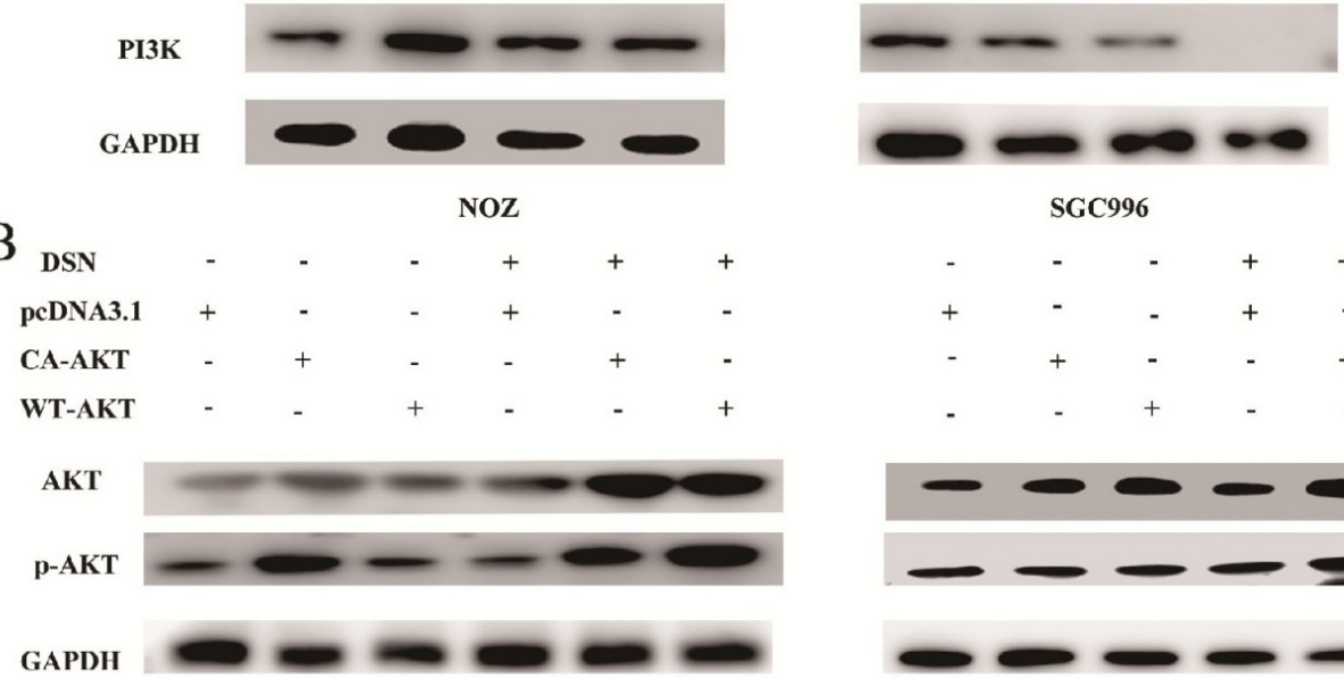

C

NOZ

D
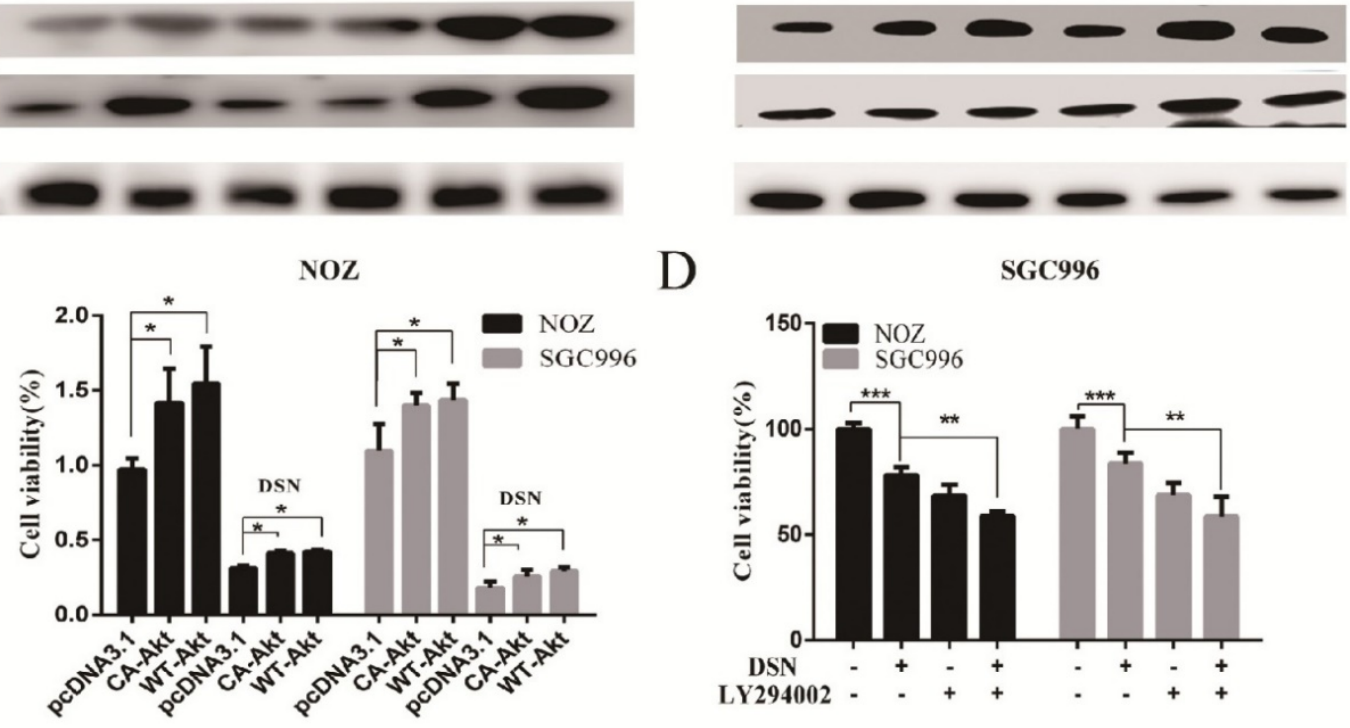

SGC996

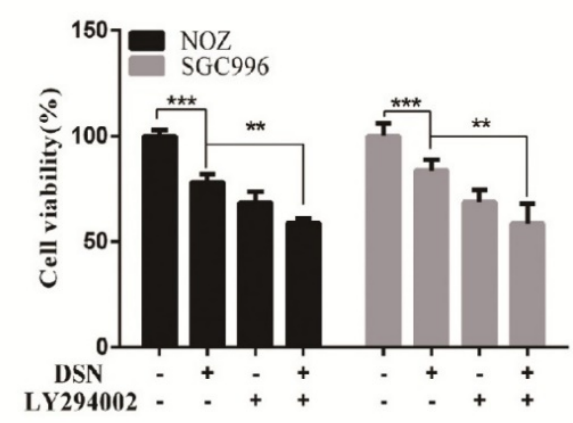

E
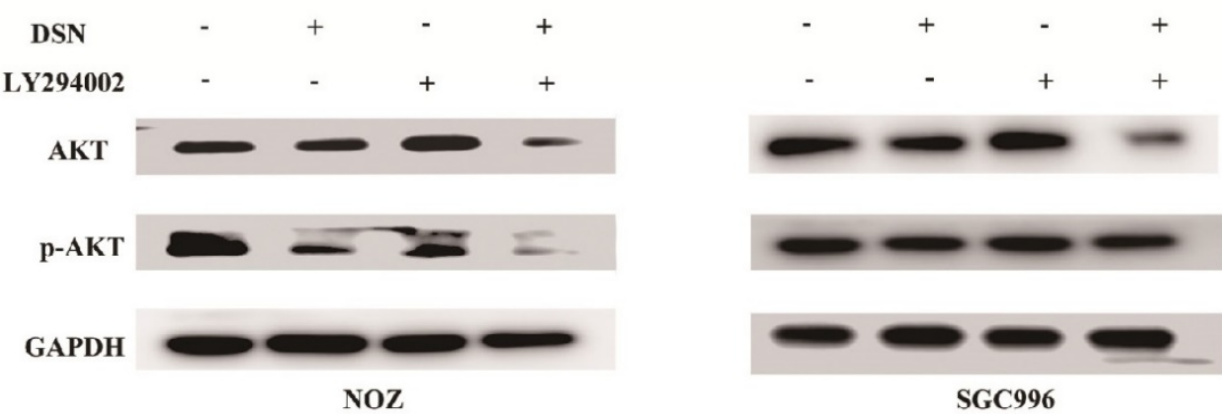

Figure 5. DSN induces GBC cell apoptosis by inhibiting the PI3K/AKT signalling pathway. A) Protein lysates from cells treated with various concentrations of DSN for $48 \mathrm{~h}$ were subjected to western blot analysisto measure AKT phosphorylation. B) AKT and p-AKT expression was analysed by western blotting after transfection with WT-AKT and CA-AKT. C) Cell viability was determined after transfection with WT-AKT and CA-AKT in the absence or presence of DSN for 24h. D) Cell

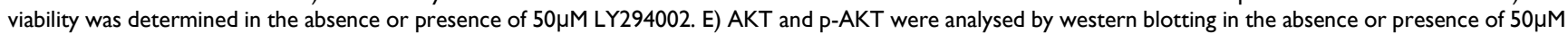
LY294002. 

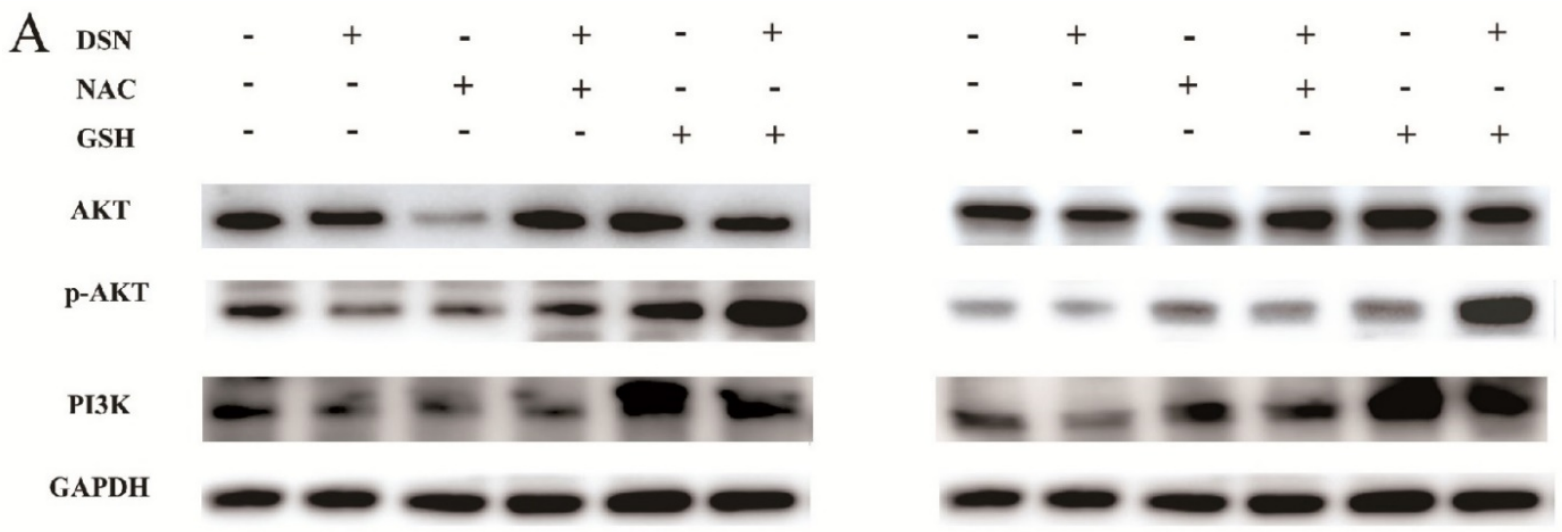

B
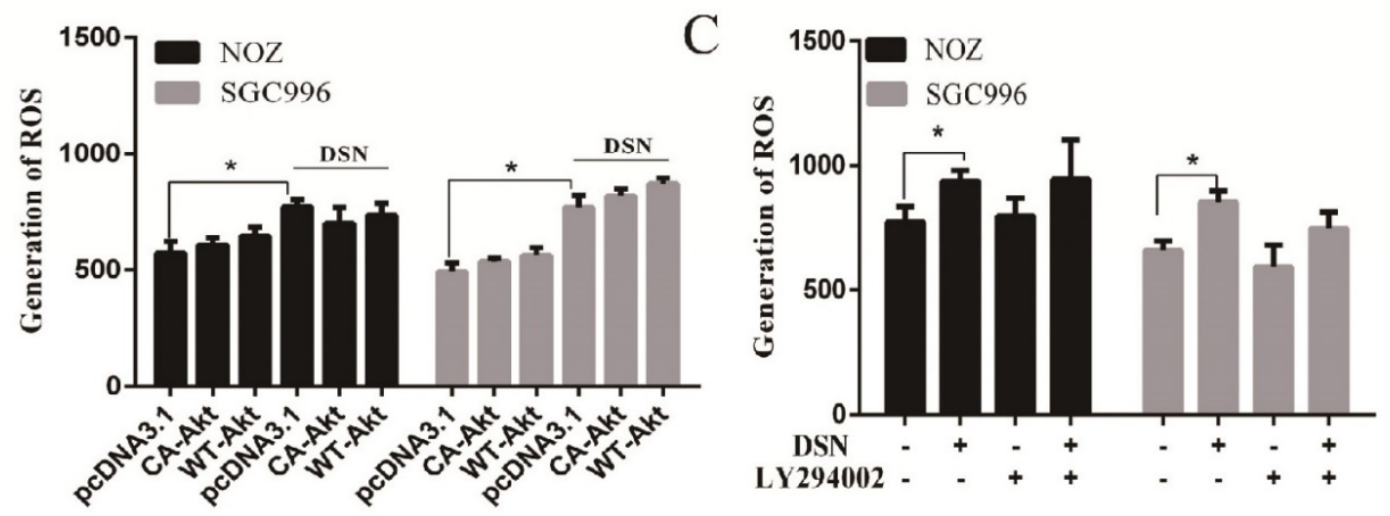

Figure 6. DSN induces GBC cell apoptosis by regulating ROS-mediated PI3K/AKT signalling. A) NOZ and SGC996 cells were treated with $4 \mu M$ DSN in the absence or presence of NAC and GSH, PI3K/AKT signaling pathway-related protein expression was analysed by western blotting. B) NOZ and SGC996 cell ROS generation after transfection with CA-AKT and WT-AKT. C) NOZ and SGC996 cell ROS generation in the absence or presence of LY294002. All data are presented as the means \pm standard deviations, and each experiment was repeated 3 times. Significant differences compared with the control are indicated by $* p<0.05$, $* * p<0.01$, and $* * * 00.001$.

\section{DSN inhibits tumour growth in vivo}

To evaluate the anti-cancer effects of DSN in vivo, we injected mice with DSN at a concentration of 0 , $5 \mathrm{mg} / \mathrm{kg}$ or $10 \mathrm{mg} / \mathrm{kg}$ every 2 days after inoculation with NOZ cells. We found that DSN inhibited tumour growth in a dose-dependent manner without other obvious appearance changes (Figure 7A- 7B, S6). Based on this observation, we performed western blot analysis, HE staining and IHC analysis. As shown in Figure 7C-7D, PI3K, p-AKT and AKT expression levels were significantly reduced compared with those of the control group. Moreover, from HE staining of the mice's liver and spleen, dioscin showed no significant effect on organ structures compared to the control group (Figure S6). These results are consistent with the in vitro effects of DSN.

\section{Discussion}

Despite significant improvements in the technical aspects of GBC detection and management, there are no known effective adjuvant therapies for GBC, although various combinations have been used clinically. The GBC metastatic cascade is complex and regulated at multiple levels. However, studies have shown that natural Chinese medicines play an important role in GBC therapy. The traditional Chinese medicine DSN has been shown to present anti-fungal, anti-virus and hepatoprotective effects. Therefore, we aimed to determine whether DSN exerts anti-cancer effects on GBC.

In this study, we investigated NOZ and SGC996 cell proliferation and viability using CCK-8 analysis and colony formation assays. As no normal gallbladder cells were available, human kidney epithelial cells (293T) was used as a control. We found that DSN significantly inhibited GBC cell proliferation, but it was not highly toxic to 293T even at higher doses $(8 \mu \mathrm{M})$. Moreover, DSN inhibited GBC cell migration at concentrations without effects of proliferation and apoptosis. Therefore, we propose that DSN represents a new and promising therapeutic agent for GBC. In the following, we evaluated the effects of DSN treatment in mice with xenografted tumours. Based on the weights and volumes, HE staining of livers and spleen of three groups, we concluded that DSN exerted anti-cancer effects on GBC cells in vivo. 

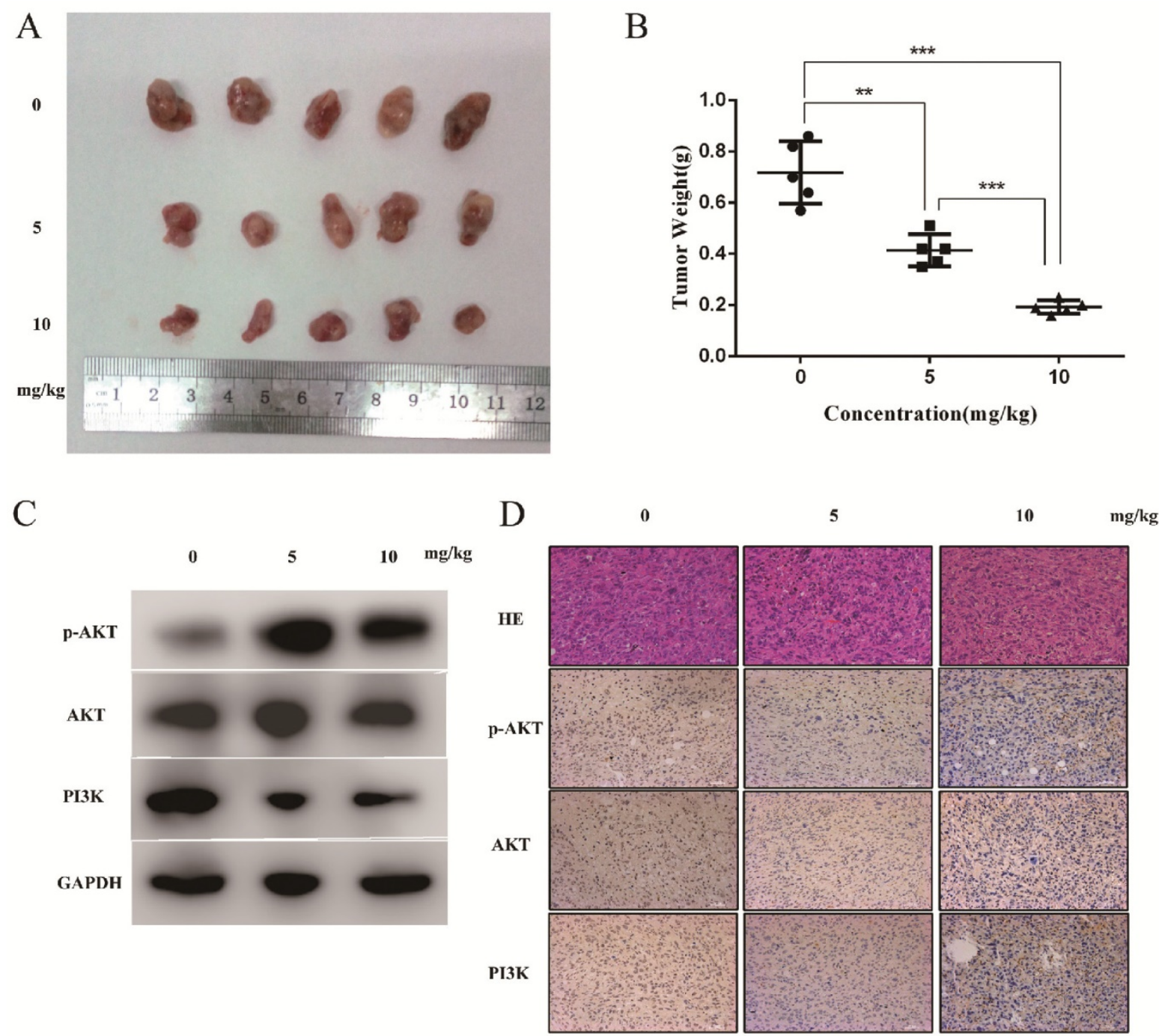

Figure 7. DSN inhibits tumour growth in vivo. A) Different concentrations $(0,5 \mathrm{mg} / \mathrm{kg}$ and $10 \mathrm{mg} / \mathrm{kg})$ of DSN were injected into nude mice after NOZ cell inoculation every 2 days. Images of 5 representative mice from each group are presented to show the sizes of the resultant tumours. B) Tumours were excised from the animals and weighed. C, D) AKT, p-AKT and PI3K expression levels were analysed using western blot analysis(C) and HE, IHC staining(D).

ROS are an important mediator of many anti-cancer agents, which are derived from at least two sources: the oxidative protein folding machinery in the endoplasmic reticulum (ER) and mitochondria. Mitochondria possess a total of nine potential ROS generation sites that can leak single electrons to oxygen and convert it into a superoxide anion, a progenitor ROS during the depletion of mitochondrial transmembrane potential $(\Delta \Psi \mathrm{m})$. Attenuation of $\Delta \Psi \mathrm{m}$ can induce caspase9 activation, which subsequently induces the caspase 3 cleavage. These caspases are believed to cause cell death through PARP cleavage, chromatin condensation, and DNA fragmentation. In our study, after treatment with different concentration of DSN for 48h, DSN-treated cells were obviously chromatin condensation and fragmentation compared with control group (Figure 2B). Moreover, we found that ROS accumulated in conjunction with $\Delta \Psi \mathrm{m}$ decrease, and the level of cleaved PARP, caspase3, 9 increased in a dose-dependent manner. Furthermore, the inhibition of apoptosis with pan-caspase inhibitor, Z-VAD-FMK, effectively blocked DSN-induced cell death, indicating that DSN induced mitochondrial dependent apoptosis.

In the following, we treated GBC cells with DSN and measured ROS generation. We found that DSN induced ROS accumulation at lower concentrations at 4h after DSN treatment. However, ROS scavengers $\mathrm{NAC}$ and GSH, can repress ROS generation and cytotoxicity, indicating that ROS was involved in DSN-induced apoptosis. GSH acts as an anti-oxidant by quenching reactive oxygen species and is involved in the ascorbate-glutathione cycle, which eliminates damaging peroxides. We measured GSH generation and noted decreased GSH depletion. Thus, we 
concluded that DSN inhibits ROS elimination and then induces significant ROS accumulation in GBC cells, leading to oxidative damage and cell death. As DSN inhibits GBC cells migration, we treated GBC cells with DSN and NAC/GSH, and found that DSN-induced migration inhibition via ROS generation. Furthermore, it is noteworthy that blocking ROS generation prevented the DSN-induced phosphorylation of PARP, caspase3 and caspase9, demonstrating that DSN stimulated the production of ROS, which subsequently actived DSN induced mitochondrial dependent apoptosis.

PI3K/AKT signalling is frequently deregulated in many human cancers, and AKT is a key downstream effector of PI3K that regulates a variety of biological processes, including survival, proliferation, apoptosis, and differentiation. Western blot analysis indicated that DSN treatment strikingly reduced PI3K/AKT pathway activation in GBC cells. Moreover, AKT and p-AKT overexpression/ inhibition abolished/enhanced DSN-induced apoptosis. However, DSN-induced migration inhibition is not related to the PI3K/AKT signalling pathway. All of these findings demonstrate that DSN inhibits GBC cell proliferation and apoptosis by regulating the PI3K-AKT signalling pathway.

Evidence indicates that transient or moderate ROS production serves as a second messenger that regulates AKT activation in various types of cells, such as haematopoietic stem cells and cardiac cells. Thus we performed experiments to confirm the relationship between ROS and the PI3K/AKT pathway. Our results showed that NAC and GSH enhanced PI3K, p-AKT and AKT expression, whereas ectopic AKT and LY294002 expression had no effect on ROS generation. Therefore, we concluded that DSN induces GBC cell apoptosis via regulating ROS-mediated PI3K/AKT signalling.

\section{Conclusion}

Taken together, these findings indicate that DSN induces GBC cell apoptosis by inhibiting of PI3K/AKT signaling through a ROS-dependent mechanism. Furthermore, DSN inhibits GBC cell migration via ROS generation. Therefore, we believe that DSN may be a novel and effective therapy for GBC.

\section{Acknowledgments}

This study was supported by the National Natural Science Foundation of China (No. 81572819,81172026, 81272402, 81301816, 81172029, 81402403, 81502433 and 31501127), China Postdoctoral Science Foundation (No. 2015M571577), the Program for Changjiang Scholars, the Natural Science Research
Foundation of Shanghai Jiao Tong University School of Medicine (No. 13XJ10037), the Leading Talent program of Shanghai and Specialized Research Foundation for the PhD Program of Higher Education-Priority Development Field (No. 20130073130014), the Interdisciplinary Program of Shanghai Jiao Tong University (No.14JCRY05), and the Shanghai Rising-Star Program (No. 15QA1403100).

\section{Supplementary Material}

Supplementary figures.

http://www.ijbs.com/v13p0782s1.pdf

\section{Abbreviations}

ROS: reactive oxygen species

GSH: glutathione

PARP: poly ADP-ribose polymerase

AKT: protein kinase $B$

p-AKT: phosphorylated protein kinase B

DMSO: dimethyl sulfoxide

CCK-8: Cell Counting Kit-8

Z-VAD-FMK: Pan-caspase inhibitor

FITC: fluorescein isothiocyanate

PI: propidium iodide

IHC: immunohistochemical streptavidin-peroxidase staining

HE: hematoxylin and eosin

SDS-PAGE: sodium dodecyl sulfate polyacrylamide gel electrophoresis

PVDF: polyvinylidene difluoride

LY294002:

2-(4-Morpholinyl)-8-phenyl-4H-1-benzopyran-4-one

PBS: phosphate buffered saline

PI3K: phosphatidylinositol 3-kinase

GAPDH: glyceraldehyde 3-phosphate dehydrogenase

\section{Competing Interests}

The authors have declared that no competing interest exists.

\section{References}

1. Li M, Zhang Z, Li X, et al. Whole-exome and targeted gene sequencing of gallbladder carcinoma identifies recurrent mutations in the ErbB pathway. Nature Genetics, 2014; 46(8):872-6.

2. Kanthan R, Senger J L, Ahmed S, et al. Gallbladder Cancer in the 21st Century. Journal of Oncology, 2015; 2015:967472.

3. Lai CHE, Lau W Y. Gallbladder cancer - A comprehensive review. Surgeon. 2008; 6(2):101-10.

4. Zhu A X, et al. Current management of gallbladder carcinoma. Oncologist, 2010;15(2):168-81.

5. Pandey M, et al. Carcinoma of the Gallbladder. Digestive Diseases \& Sciences, 2001;46(6):1145-51.

6. $\mathrm{Li} \mathrm{M}$, et al. Yes-associated protein 1 (YAP1) promotes human gallbladder tumor growth via activation of the AXL/MAPK pathway. Cancer Lett, 2014;355(2):201-9.

7. Shu Y J, et al. SPOCK1 as a potential cancer prognostic marker promotes the proliferation and metastasis of gallbladder cancer cells by activating the PI3K/AKT pathway. Mol Cancer, 2015;14:12.

8. Subramani R, et al. Nimbolide inhibits pancreatic cancer growth and metastasis through ROS-mediated apoptosis and inhibition of epithelial-to-mesenchymal transition. Sci Rep, 2016;6:19819. 
9. Hsieh M J, et al. Dioscin-induced autophagy mitigates cell apoptosis through modulation of PI3K/Akt and ERK and JNK signaling pathways in human lung cancer cell lines. Arch Toxicol, 2013;87(11):1927-37.

10. Wang Y, et al. Dioscin (Saponin)-Induced Generation of Reactive Oxygen Species through Mitochondria Dysfunction: A Proteomic-Based Study. J Proteome Res, 2007;6(12):4703-10.

11. Tong $\mathrm{Q}$, et al. Dioscin inhibits colon tumor growth and tumor angiogenesis through regulating VEGFR2 and AKT/MAPK signaling pathways. Toxicol Appl Pharm, 2014;281(2):166-73.

12. Kim EA, et al. Dioscin induces caspase-independent apoptosis through activation of apoptosis-inducing factor in breast cancer cells. Apoptosis, 2014;19(7):1165-75.

13. Zhao $X$, et al. Potent effects of dioscin against gastric cancer in vitro and in vivo. Phytomedicine. 2016;23(3):274-82.

14. Titus E A. Degradation of Misfolded Proteins Prevents ER-Derived Oxidative Stress and Cell Death. Mol Cell, 2004;15(5):767-76.

15. Trachootham D, Alexandre J, Huang P. Targeting cancer cells by ROS-mediated mechanisms: a radical therapeutic approach? Nat Rev Drug Discov, 2009;8(7):579-91.

16. Gong K, Li W. Shikonin, a Chinese plant-derived naphthoquinone, induces apoptosis in hepatocellular carcinoma cells through reactive oxygen species: A potential new treatment for hepatocellular carcinoma. Free Radical Bio Med, 2011;51(12):2259-71.

17. Sabharwal S S, Schumacker P T. Mitochondrial ROS in cancer: initiators, amplifiers or an Achilles' heel? Nat Rev Cancer, 2014;14(11):709-21.

18. Hengartner M O. The biochemistry of apoptosis. Nature, 2000;407(6805):770-6.

19. Jacobson M D. Reactive oxygen species and programmed cell death. Trends Biochem Sci, 1996;21(3):83-6.

20. Meng Q, et al. Role of PI3K and AKT specific isoforms in ovarian cancer cell migration, invasion and proliferation through the p70S6K1 pathway. Cell Signal. 2006;18(12):2262-71.

21. Chan E, Berlin J. Biliary Tract Cancers: Understudied and Poorly Understood. J Clin Oncol, 2015;33(16):1845-8

22. Jiang L, et al. Bufalin induces cell cycle arrest and apoptosis in gallbladder carcinoma cells. Tumour Biol, 2014;35(11):10931-41.

23. Maolan $\mathrm{Li}$, et al. Magnolol inhibits growth of gallbladder cancer cells through the p53 pathway. Cancer Sci, 2015;106(10):1341-50.

24. Sena L, Chandel N. Physiological Roles of Mitochondrial Reactive Oxygen Species. Mol Cell, 2012;48(2):158-67.

25. Qiyan Cai, Teng Ma, Chengren Li, et al. Catalpol Protects Pre-Myelinating Oligodendrocytes against Ischemia-induced Oxidative Injury through ERK1/2 Signaling Pathway. Int. J. Biol.Sci.2016; 12(12): 1415-1426.

26. Guzy RD, et al. Mitochondrial complex III is required for hypoxia-induced ROS production and cellular oxygen sensing. Cell Metab, 2005;1(6):401-8.

27. D'Autréaux B, Toledano M B. ROS as signalling molecules: mechanisms that generate specificity in ROS homeostasis. Nat Rev Mol Cell Bio. 2007;8(10):813-24.

28. Fulda S. Targeting apoptosis for anticancer therapy. Semin Cancer Biol. 2015;31:84-8.

29. Deepak M. Kasote, Surendra S. Katyare, Mahabaleshwar V. Hegde, et al. Significance of Antioxidant Potential of Plants and its Relevance to Therapeutic Applications. Int J Biol Sci. 2015; 11(8): 982-991.

30. Fresno Vara, et al. PI3K/Akt signalling pathway and cancer. Cancer Treat. 2004;30(2):193-204

31. Yuzhen $\mathrm{Yao}$, et al. a-Lipoic acid increases tolerance of cardiomyoblasts to glucose/glucose oxidase-induced injury via ROS-dependent ERK1/2 activation. Biochimica Et Biophysica Acta, 2012;1823(4):920-9.

32. Surong Jiang, et al. a-Lipoic acid protected cardiomyoblasts from the injury induced by sodium nitroprusside through ROS-mediated Akt/Gsk-3 $\beta$ activation. Toxicology in Vitro, 2014;28(8):1461-73. 